$\underline{\text { Preprint typeset in JHEP style. - HYPER VERSION }}$

ROM2F-2000-33

\title{
RG-flows and Open/Closed String Duality
}

\author{
Massimo Bianchi and Jose F. Morales \\ Dipartimento di Fisica, Università di Roma "Tor Vergata" \\ I.N.F.N. - Sezione di Roma "Tor Vergata" \\ Via della Ricerca Scientifica, 1 \\ 00173 Roma, ITALY
}

\begin{abstract}
We discuss the interpaly between IR and UV divergences in theories with open and unoriented strings in view of the AdS/CFT correspondence. We start by deriving general formulas for the computation of threshold corrections to gauge couplings in generic configurations with open and unoriented strings. These allow us to discuss the IR/UV correspondence between beta-function coefficients and "dilaton" tadpoles for several brane configurations probed by D3-branes. Finally we comment on the AdS supergravity descriptions of gauge theories that are (super)conformal in the large $N$ limit.
\end{abstract}

KEYwORDS: RG-flows, tadpoles, open strings. 


\section{Contents}

1. Introduction 1

2. Infrared/Ultraviolet divergences in open string amplitudes 3

2.1 "BPS saturated" string amplitudes 8

3. D3-branes probes in type II and type IB backgrounds 11

3.1 D3-branes probing type II D7-backgrounds 12

3.2 D3-branes in type IB backgrounds 13

3.3 D3-branes probes of a $C^{4} / Z_{2}$ Orientifold singularity 14

3.4 D3-branes probes of a $C^{6} / Z_{2} \times Z_{2}$ Orientifold singularity

4. AdS/CFT correspondence and final comments 16

\section{Introduction}

The interplay between open and closed string divergences in amplitudes around nontrivial configurations with Dirichlet branes and orientifold planes is of particular import to all recent developments in string theory.

On the one hand, it has been recently shown in [1] that on very general grounds closed string divergences associated to massless R-R fields disappearing into the vacuum (R-R tadpoles) are always associated to anomalies in the open-string theory that describe the excitations of the brane background. On the other hand, NS-NS tadpoles, such as the dilaton tadpole, do not necessarily imply a pathology of the theory rather they signal an instability of the vacuum configuration one is expanding around. Long time ago Fischler and Susskind have proposed an algorithm for systematically reabsorbing such divergences [2]. More recently the role of the dilaton tadpole has been analyzed in connection to the AdS/CFT correspondence [3]. Interestingly the dilaton tadpole on the disk and projective plane for some non-tachyonic unoriented projections [4] of the type OB theory have been shown to be in quantitative agreement with the one-loop $\beta$-function coefficient of the four dimensional gauge theory living on D3-brane probes [3, 5]. Given the standard relation between ten dimensional dilaton and gauge coupling on the brane it is natural to ask to what extent the relation found in [3] generalizes to other configurations with open and unoriented strings. 
In this respect, it is convienient to observe that up to some "innocuous" Tduality transformation, vacuum configurations with open and unoriented strings can be related to F-theory backgrounds or more efficiently configurations with D7-branes and O7-planes wrapping possibly non-trivial cycles. Most of the emphasis so far has been on the configurations with constant dilaton that can pe probed by D3-branes giving rise to interesting (super)conformal field theories [6]. Non-conformal gauge theories arising on D3-brane probes of tachyonic type O backgrounds have been studied [7, 8]. Some RG-flows obtained by perturbing superconformal fixed points by relevant operators have been studied by various groups [9, 10, 11]. Although the five-dimensional perspective allows one to derive a holographic c-theorem and in principle to compute mass spectra and correlation functions in the boundary gauge theory [12 a lift to ten dimensions seems to be required in order to understand the fate of some curvature singularities [13] and the running of the gauge coupling [14]. Related work on the subject also appeared in [15].

Our emphasis will be on theories that arise from a (super-)conformal background by displacing some of the branes in such a way that the 10-D dilaton tadpole does not vanish locally. Following [3] we expect that the resulting gauge theory on the brane should display a running coupling with a one-loop coefficient related to the dilaton tadpole on the disk/projective plane. We will find that the quantitative agreement between dilaton tadpole and $\beta$-function coefficient can be substantiated for sectors of the open-string spectrum that enjoy $\mathcal{N}=2$ supersymmetry (i.e. 8 supercharges). The resulting theory need not necessarily be supersymmetric because the supercharges preserved in one sector can be different from the supercharges preserved in another sector. We will argue that this underlying "fictitious" $\mathcal{N}=2$ supersymmetry protects the IR/UV correspondence between tadpoles and RG-flows from higher loop corrections at the leading order in the 't Hooft coupling $\hat{g}^{2}=g^{2} N$ for all $\mathcal{N}=1,2$ examples presented here. This is not the case for the $\mathcal{N}=0$ examples presented in [3] for which the correspondence still works at the one-loop level but is expected to receive non-trivial corrections at higher orders both in the SYM and in the Supergravity descriptions. A quantitative relation between "properly regularized" dilaton tadpole at one-loop (annulus, ....) and two-loop coefficient of the $\beta$ function would be of particular significance but very hard to establish because of the well-known ambiguities in superstring perturbation theory beyond one-loop.

The plan of the paper is as follows. In Section 2 we discuss divergences in string amplitudes in the presence of non-trivial backgrounds for the boundary (gauge) fields. Closely following [16, 17], we derive general formulas for the computation of threshold corrections to gauge couplings in configurations with open and unoriented strings. The results apply to any configuration of open strings satisfying the basic consistency requirements developed in [18, 19]. In Section 3 we discuss the IR/UV correspondence between $\beta$-function coefficients and "dilaton" tadpoles for several examples of brane configurations constructed in terms of D3-branes, D7-branes and 
orientifold planes. In Section 4 we comment on the AdS supergravity descriptions of gauge theories that are (super)conformal in the large $N$ limit. In particular we show how the subleading logarithmic running of the gauge coupling is quantitatively reproduced in the holographic correspondence by the evolution of the 10-D dilaton caused by the presence of non-vanishing tadpoles on the disk and projective plane.

\section{Infrared/Ultraviolet divergences in open string amplitudes}

In this section we apply the techniques developed in [16, 17] to the study of the infrared/ultraviolet divergences of one-loop open string partition functions in the presence of non-trivial backgrounds for the boundary gauge fields.

String amplitudes of this kind are efficiently encoded in helicity supertraces over the Hilbert space of open string states connecting different boundaries and crosscaps. The Hilbert space of open string states can be decomposed into a (generically infinite) sum $\mathcal{H}=\oplus_{i} \mathcal{H}_{i}$ of Verma moduli labeled by the primary fields $\Phi^{i}$ of some chiral algebra $\mathcal{G}$, that includes an $\mathcal{N}=1$ superconformal algebra at least. The subspace $\mathcal{H}_{i}$ consists in the tower of descendants under $\mathcal{G}$ of the groundstate $\left|\Delta_{i}\right\rangle=\Phi^{i}|0\rangle$. The helicity supertrace over states in $\mathcal{H}_{i}$ is defined by the holomorphic elliptic function

$$
\mathcal{X}_{i}(u \mid \tau)=\operatorname{STr}_{\mathcal{H}_{i}}^{\prime}\left[q^{L_{0}-\frac{c}{24}} e^{2 \pi i u_{\mu \nu} J^{\mu \nu}}\right],
$$

with $L_{0}$ the Virasoro generator (open string Hamiltonian), $J^{\mu \nu}$ the generators of the $S O(D-2)$ (little) Lorentz group and $q=e^{2 \pi i \tau}$. A prime in (2.1) indicates the omission of the bosonic zero-modes contribution to the trace, that will be explicitly displayed in the next formulas. For "rectangular" toroidal compactifications, i.e. for vanishing off-diagonal components of the metric and B-field, it is given by

$$
\frac{1}{t^{\frac{D}{2}}} \Sigma_{\Lambda}(t) \equiv \frac{1}{t^{\frac{D}{2}}} P_{n_{\Lambda}}(t) W_{d_{\Lambda}}(t)=\frac{1}{t^{\frac{D}{2}}} \sum_{\left(m_{i}, n_{j}\right) \in \mathbf{Z}^{n_{\Lambda}+d_{\Lambda}}} e^{-\pi t\left(\frac{\alpha^{\prime} m_{i}^{2}}{R_{i}^{2}}+\frac{n_{j}^{2} R_{j}^{2}}{\alpha^{\prime}}\right)}
$$

where $D$ is the number of non-compact directions ${ }^{1}, P_{n_{\Lambda}}(t)\left(W_{d_{\Lambda}}(t)\right)$ are $n_{\Lambda}\left(d_{\Lambda}\right)$ dimensional momentum(winding) lattice sums for open strings with NN(DD) boundary conditions labeled by the lattice $\Lambda$ that defines the torus $T^{n_{\Lambda}+d_{\Lambda}}=R^{n_{\Lambda}+d_{\Lambda}} / \Lambda$.

Modular invariance (of the bulk theory) ensures that helicity supertraces (2.3) can be written as sums over even spin structures ${ }^{2}$

$$
\mathcal{X}_{i}(u \mid \tau)=\sum_{(\alpha, \beta) \neq\left(\frac{1}{2}, \frac{1}{2}\right)} \mathcal{X}_{i}\left[\begin{array}{l}
\alpha \\
\beta
\end{array}\right](\tau) \mathcal{F}\left[\begin{array}{c}
\alpha \\
\beta
\end{array}\right](u \mid \tau)=h^{i}(u)+O(q)
$$

\footnotetext{
${ }^{1}$ The resulting hyperplane is to be identified with the spacetime under consideration. We assume NN boundary conditions along it for all open string excitations of the brane configuration.

${ }^{2}$ The odd spin structure contribution, which is crucial in establishing the relation between R-R tadpoles and anomalies, will play no role in the thresholds we consider hereafter.
} 
with $\mathcal{X}_{i}\left[\begin{array}{l}\alpha \\ \beta\end{array}\right](\tau)$ the contribution of the sector with $(\alpha, \beta)$ spin structure to the open string partition function and

$$
\mathcal{F}\left[\begin{array}{l}
\alpha \\
\beta
\end{array}\right](u \mid \tau)=\prod_{m=1}^{\frac{D-2}{2}} \frac{\vartheta\left[\begin{array}{l}
\alpha \\
\beta
\end{array}\right]\left(u_{m} \mid \tau\right)}{\vartheta\left[\begin{array}{l}
\alpha \\
\beta
\end{array}\right](0 \mid \tau)} \frac{\vartheta\left[\begin{array}{c}
\frac{1}{2} \\
\frac{1}{2}
\end{array}\right]^{\prime}(0 \mid \tau)}{\vartheta\left[\begin{array}{l}
\frac{1}{2} \\
\frac{1}{2}
\end{array}\right]\left(u_{m} \mid \tau\right)} u_{m}
$$

is the spacetime helicity supertrace which has been normalized to one for $u=0$. Without loss of generality $u_{\mu \nu}$ has been taken in the block diagonal form $u=$ $u_{m} \delta_{2 m, 2 m-1}$. In the right hand side of (2.3) we have assumed that the theories we deal with are free of tachyons. For later convenience we have introduced the notation $h^{i}(u)$ for the helicity supertrace restricted to massless states in $\mathcal{H}_{i}$. It can be written as

$$
h_{i}(u)=\sum_{r} h_{i}^{(r)} u^{r}=\operatorname{Str}_{\mathcal{H}_{i}}^{\text {massless }} \prod_{m}\left[\frac{\pi u_{m}}{\sin \left(\pi u_{m}\right)}\right] \sum_{m} \cos \left(2 \pi u_{m} J_{m}\right)
$$

with $J_{m}$ the eigenvalue of the helicity operator in the $(2 m, 2 m-1)$ plane.

The modular transformation properties of the character-valued partition functions $\mathcal{X}_{i}(u \mid \tau)$ are determined by the modular transformation properties of the characters. Denoting by $S_{i}^{j}$ and $T_{i}^{j}$ the matrices representing the generators of the modular group in the $\mathcal{X}_{i}(0 \mid \tau)$ basis, one finds

$$
\begin{aligned}
T: & \mathcal{X}_{i}(u \mid \tau+1) & =e^{2 \pi i\left(\Delta_{i}-c / 24\right)} \mathcal{X}_{i}(u \mid \tau) \\
S: & \mathcal{X}_{i}\left(\frac{u}{\tau} \mid-\frac{1}{\tau}\right) & =(i \tau)^{-\frac{D+n+d-2}{2}} S_{i}^{j} \mathcal{X}_{j}(u \mid \tau)
\end{aligned}
$$

where $\Delta_{i}$ is the conformal dimension of the primary field $\Phi_{i}$. Hereafter a sum over repeated indices is always understood: $a^{i} b^{i} c^{i} \ldots \equiv \sum_{i} a^{i} b^{i} c^{i} \ldots$

Following [20, 21] one can write the open string partition function in the presence of a constant electromagnetic background $F_{12}=f Q$, with $Q$ a generator in the Cartan-subalgebra normalized in such a way that $\operatorname{tr}_{\text {fund }} Q^{2}=\frac{1}{2}$. The net result of the non-trivial background is a shift of the oscillator frequencies of the complex coordinate $X^{1}+i X^{2}$ by an amount $\epsilon$ given by

$$
\pi \epsilon_{a b}=\arctan \left(\pi f q_{a}\right)+\arctan \left(\pi f q_{b}\right)
$$

with $q_{a}, q_{b}$ the charges of the boundaries $a, b$ respectively where the open string ends (for the Möbius strip $q_{a}=q_{b}$ ). Generalizing [16, 17], the (direct channel) partition function for the open string excitations of a brane configuration in the presence of an electromagnetic background can be written as

$$
\begin{aligned}
\mathcal{A}(f) & =\frac{1}{2} A_{a b}^{i \Lambda} \operatorname{Tr}_{n^{a} \otimes n^{b}} \frac{\pi f\left(q_{a}+q_{b}\right)}{\epsilon_{a b}} \int \frac{d t}{t^{1+\frac{D}{2}}} \mathcal{X}_{i}\left(\epsilon_{a b} \frac{i t}{2} \mid \frac{i t}{2}\right) \Sigma_{\Lambda}(t) \\
\mathcal{M}(f) & =\frac{1}{2} M_{a}^{i \Lambda} \operatorname{Tr}_{n^{a}} \frac{2 \pi f q_{a}}{\epsilon_{a a}} \int \frac{d t}{t^{1+\frac{D}{2}}} \widehat{\mathcal{X}_{i}}\left(\epsilon_{a a} \frac{i t}{2} \mid \frac{i t}{2}+\frac{1}{2}\right) \Sigma_{\Lambda}(t)
\end{aligned}
$$


with the integers $A_{a b}^{i \Lambda}$ and $M_{a}^{i \Lambda}$ counting the number of times the open string sector $i$ with internal momenta belonging to the lattice $\Lambda$ and ends of type $(a, b)$ and $(a, a)$ runs along the Annulus and Möbius-strip loop, respectively. Alternatively, after the change of variables $\ell_{A}=\frac{2}{t}$ and $\ell_{M}=\frac{1}{2 t}$ in (2.8), one can reinterpret these amplitudes as tree level exchange (transverse channel) of closed string states between the boundaries and crosscaps

$$
\begin{aligned}
\mathcal{A}(f) & =\frac{2}{2}{ }^{-\frac{D+n+d}{2}} B_{a}^{i \Lambda} B_{b}^{i \Lambda} \operatorname{Tr}_{n^{a} \otimes n^{b}} \frac{\pi f\left(q_{a}+q_{b}\right)}{\epsilon_{a b}} \int d \ell \mathcal{X}_{i}\left(\epsilon_{a b} \mid i \ell\right) \Sigma_{\Lambda}\left(\frac{\ell}{2}\right) \\
\mathcal{M}(f) & =\frac{2}{2} \Gamma^{i \Lambda} B_{a}^{i \Lambda} \operatorname{Tr}_{n^{a}} \frac{2 \pi f q_{a}}{\epsilon_{a a}} \int d \ell \widehat{\mathcal{X}_{i}}\left(\frac{\epsilon_{a a}}{2} \mid i \ell+\frac{1}{2}\right) \Sigma_{\Lambda}^{\mathrm{e}}\left(\frac{\ell}{2}\right)
\end{aligned}
$$

$\Gamma^{i \Lambda}$ and $B_{a}^{i \Lambda}$ are the reflection coefficients (one-point function) of a state in the character " $i$ " with internal momentum in $\Lambda$ in front of a crosscap or a boundary of type $a$, respectively. They are related to the integer coefficients in the direct channel by suitable modular transformations:

$$
\begin{aligned}
A_{a b}^{i \Lambda} & =S_{i^{\prime}}^{i} s_{\Lambda^{\prime}}^{\Lambda} B_{a}^{i^{\prime} \Lambda^{\prime}} B_{b}^{i^{\prime} \Lambda^{\prime}} \\
M_{a}^{i \Lambda} & =P_{i^{\prime}}^{i} s_{\Lambda^{\prime}}^{\Lambda} \Gamma^{i^{\prime} \Lambda^{\prime}} B_{a}^{i^{\prime} \Lambda^{\prime}}
\end{aligned}
$$

with $S_{i^{\prime}}^{i}$ defined in (2.6), $P \equiv T^{1 / 2} S T^{2} S T^{1 / 2}$ and $s_{\Lambda^{\prime}}^{\Lambda}$ the result of the Poisson resummation

$$
\Sigma_{\Lambda}\left(\frac{1}{t}\right)=P_{n_{\Lambda}}\left(\frac{1}{t}\right) W_{d_{\Lambda}}\left(\frac{1}{t}\right)=t^{-\frac{n_{\Lambda}+d_{\Lambda}}{2}} s_{\Lambda^{\prime}}^{\Lambda} \Sigma_{\Lambda^{\prime}}(t)=t^{-\frac{n_{\Lambda}+d_{\Lambda}}{2}} \frac{v_{n_{\Lambda}}}{v_{d_{\Lambda}}} W_{n_{\Lambda}}(t) P_{d_{\Lambda}}(t)
$$

$v_{n_{\Lambda}}, v_{d_{\Lambda}}$ are the volumes of the NN, DD compact hyperplanes (labeled by $\Lambda$ ) measured in units of $2 \pi \alpha^{\prime}$. Finally the notation $\Sigma_{\Lambda}^{\mathrm{e}}(\ell)$ in the transverse Möbius amplitude denotes a restriction to even integers in the lattice sum, i.e. to the states that can be reflected by a crosscap [22, 23].

One-loop open string amplitudes involving an arbitrary number of boundary field insertions $F_{12}$ can be derived from (2.8) by expanding to a suitable order in the field strength background $f$. In general they suffer from both infrared and ultraviolet divergences. Introducing in (2.8,2.9) $\lambda_{I R}\left(\lambda_{U V}\right)$ cutoff's for the infrared(ultraviolet) divergences one is left with the regularized modular integrals

$$
\mathcal{A}(f)+\mathcal{M}(f)=\int_{2 \lambda_{U V}}^{2 \lambda_{I R}} \frac{d t}{t} A(f \mid t)+\int_{\frac{\lambda_{U V}}{2}}^{\frac{\lambda_{I R}}{t}} \frac{d t}{t} M(f \mid t)=\int_{\lambda_{I R}^{-1}}^{\lambda_{U V}^{-1}} d \ell[\widetilde{A}(f \mid \ell)+\widetilde{M}(f \mid \ell)] .
$$

Following [16, 17] we have choosen to work with a uniform cutoff in the transverse channel that give rise to non-uniform cutoffs in the direct channel.

The (divergent) coupling constants of the operators $\operatorname{Tr}_{\mathcal{R}} \mathcal{F}^{r}$ ( $\mathcal{R}$ running over the spectrum of open string representations) are compactly encoded in the coefficients 
of the expansion in powers of $f$ of "generating $\beta^{a}(f)$ functions" defined by

$$
\begin{aligned}
\beta_{I R}(f) \equiv & \sum_{r} \beta_{I R}^{a(r)} \operatorname{Tr}_{n_{a}}\left(\pi f q_{a}\right)^{r} \equiv \lambda_{I R} \frac{\partial}{\partial \lambda_{I R}}\left[\int_{2 \lambda_{U V}}^{2 \lambda_{I R}} \frac{d t}{t} A(f \mid t)+\int_{\frac{\lambda_{U V}}{2}}^{\frac{\lambda_{I R}}{2}} \frac{d t}{t} M(f \mid t)\right] \\
& =\lim _{\lambda_{I R} \rightarrow \infty}\left[A\left(f \mid 2 \lambda_{I R}\right)+M\left(f \mid \frac{\lambda_{I R}}{2}\right)\right] \\
\beta_{U V}(f) \equiv & \sum_{r} \beta_{U V}^{a(r)} \operatorname{Tr}_{n_{a}}\left(\pi f q_{a}\right)^{r} \equiv-\lambda_{U V} \frac{\partial}{\partial \lambda_{U V}} \int_{\lambda_{I R}^{-1}}^{\lambda_{U V}^{-1}} d \ell[\widetilde{A}(f \mid \ell)+\widetilde{M}(f \mid \ell)] \\
& =\lim _{\lambda_{U V} \rightarrow 0} \lambda_{U V}^{-1}\left[\widetilde{A}\left(f \mid \lambda_{U V}^{-1}\right)+\widetilde{M}\left(f \mid \lambda_{U V}^{-1}\right)\right]
\end{aligned}
$$

Infrared divergences in (2.13) are associated to the running of the gauge couplings in the effective gauge theory living on the branes. Ultraviolet divergences arise from the factorization of the transverse amplitudes into the disk and projective plane and are associated to the infinite time propagation of massless supergravity states. Both kinds of divergences receive only contributions from massless states in the relevant description and therefore can be studied using an effective Supergravity (bulk theory) or SYM (brane theory) picture according to the case. The answers disagree in general but under special circumstances they lead to the same result. The main aim of this paper is to explore branches in the moduli space of certain brane configurations where RG-flows in the "boundary" gauge theory receive an accurate description in terms of the "bulk" supergravity theory.

We will concentrate our attention in RG-flows of the irreducible couplings $\operatorname{Tr}_{n_{a}} \mathcal{F}^{r}$ in the gauge theory. Plugging (2.8) in (2.13) and noticing that in the limit $t \rightarrow \infty$ the leading contributions (at a given order $r$ ) comes from the expansion of $\mathcal{X}_{i}$ in its first argument, one can simply replace $\epsilon_{a b}$ with $\pi f\left(q_{a}+q_{b}\right)$. One is then left with the $\beta$-function coefficients

$$
\beta_{I R}^{a(r)}=\frac{h_{i}^{(r)}}{2}\left[2^{1-\frac{D}{2}} A_{a b}^{i \Lambda} n^{b} \Sigma_{\Lambda}\left(2 \lambda_{I R}\right)+2^{\frac{D}{2}-r} M_{a}^{i \Lambda} \Sigma_{\Lambda}\left(\frac{\lambda_{I R}}{2}\right)\right] \lambda_{I R}^{r-\frac{D}{2}}
$$

with $h_{i}^{(r)}$ the massless helicity supertrace coefficients defined through (2.5). In particular, after expanding (2.5) one gets for the first few coefficients

$$
h_{i}(u)=\sum_{r} h_{i}^{(r)} u^{r}=\operatorname{Str}_{i} 1+u^{2} \operatorname{Str}_{i}\left(-2 J^{2}+\frac{1}{6}\right)+\frac{u^{4}}{3} \operatorname{Str}_{i}\left(2 J^{4}-J^{2}+\frac{7}{120}\right)+\ldots
$$

with $J=J_{12}$ the (eigenvalue of the) helicity operator in the (12)-plane. Evaluating the helicity traces (2.16) yields

$$
\begin{aligned}
& h_{i}^{(0)}=2 n_{V}^{i}+n_{O}^{i}-\left(n_{S}^{i}+n_{C}^{i}\right) \\
& h_{i}^{(2)}=-\frac{11}{3} n_{V}^{i}+\frac{1}{6} n_{O}^{i}+\frac{2}{3}\left(n_{S}^{i}+n_{C}^{i}\right) \\
& h_{i}^{(4)}=\frac{1}{360}\left[254 n_{V}^{i}+7 n_{O}^{i}+16\left(n_{S}^{i}+n_{C}^{i}\right)\right]
\end{aligned}
$$


where $n_{O}^{i}, n_{V}^{i}, n_{S}^{i}$ and $n_{C}^{i}$ are integers counting the number of massless states in the character " $i$ " transforming as scalars, vectors, left- and right-handed spinors, respectively, i.e. having helicity $J=0, J= \pm 1$, and $J= \pm \frac{1}{2}$. One recognizes in $h_{i}^{(2)}$ the familiar $\beta$-function coefficients associated to the running of the $\mathcal{F}^{2}$ term in four dimensional gauge theories. To this end one should recall that $n_{S}^{i}=n_{C}^{i}=n_{F}^{i} / 2$ as a consequence of the CPT theorem in $D=4$. Much in the same way, $h_{i}^{(0)}$ and $h_{i}^{(4)}$ can be associated to the logarithmically running self-energy (0-point function) in $D=0$ and four-point coupling $\operatorname{Tr}_{\mathcal{R}} \mathcal{F}^{4}$ in $D=8$.

A similar analysis can be performed in the study of ultraviolet divergences of open string partition functions in an electromagnetic background. After some simple trigonometric manipulations one finds ${ }^{3}$

$$
\begin{aligned}
\widetilde{h}_{i}(f)= & h_{i}\left(\epsilon_{a b}\right) \frac{\pi f\left(q_{a}+q_{b}\right)}{\epsilon_{a b}} \\
= & \frac{\pi f\left(q_{a}+q_{b}\right)}{\sin \left(\pi \epsilon_{a b}\right)}\left[n_{0}^{i}+2 \cos \left(2 \pi \epsilon_{a b}\right) n_{V}^{i}-\cos \left(\pi \epsilon_{a b}\right)\left(n_{S}^{i}+n_{C}^{i}\right)\right] \\
= & \frac{1}{\sqrt{1+\pi^{2} f^{2} q_{a}^{2}} \sqrt{1+\pi^{2} f^{2} q_{b}^{2}}}\left[\left(n_{0}^{i}+2 n_{V}^{i}\right)\left(1+\pi^{2} f^{2} q_{a}^{2}\right)\left(1+\pi^{2} f^{2} q_{b}^{2}\right)\right. \\
& \left.-4 n_{V}^{i} \pi^{2} f^{2}\left(q_{a}+q_{b}\right)^{2}\right]-\left(n_{S}^{i}+n_{C}^{i}\right)\left(1-q_{a} q_{b} \pi^{2} f^{2}\right)
\end{aligned}
$$

By inspection of (2.9) one sees that Annulus and Möbius amplitudes factorize into two disks or a disk and a crosscap with certain number of insertions of gauge fields on the boundaries. It is remarkable that for any consistent open-string descendant (even without a clear geometrical meaning) one can deduce generalized Born-Infeld and WZ couplings much in the same way as in 24. From the factorization of (2.18) one can extract the coupling of the brane theory to the bulk NS-NS scalars $\left(n_{O}\right)$, graviton and dilaton $\left(n_{V}\right)$ and R-R tensor fields $\left(n_{S}, n_{C}\right)$. For flat D-branes this amounts to the familiar fact that (2.18) can be derived from the effective worldvolume action

$$
S_{\text {brane }}=S_{B I}+S_{W Z}
$$

with

$$
\begin{aligned}
S_{B I}= & \int d^{p+1} e^{-\phi} \sqrt{\operatorname{det}(\mathcal{G}+\mathcal{F})} \\
& =\frac{e^{-\phi_{0}}}{4} \sqrt{1+\pi^{2} f^{2} q^{2}}\left(1+\frac{1}{4} h_{\mu \mu}-\frac{1}{4} h_{i i}+\frac{2 \pi f b_{12}-\pi^{2} f^{2} q^{2}\left(h_{11}+h_{22}\right)}{2\left(1+\pi^{2} f^{2} q^{2}\right)}+\ldots\right) \\
S_{W Z}= & \int\left(C_{p+1}+C_{p-1} \wedge \mathcal{F}\right)
\end{aligned}
$$

with $h^{\mu \mu}, h_{i i}$ the metric components along and transverse to the brane respectively, $b_{12}$ the NS-NS $B$-field fluctuation, $F_{12}=\pi q f$ and $\phi=\frac{1}{4}\left(h_{\alpha}^{\alpha}+h_{i}^{i}\right)$ the ten dimensional

\footnotetext{
${ }^{3}$ The discrepancies in signs with respect to [24] is due to our choice (as in [16]) of defining the charges at the two ends of the string without a relative minus sign.
} 
dilaton. The dots in (2.20) represent higher order powers of the fluctuations $h_{M N}$, $b_{M N}$ in the expansions $G_{M N}=\eta_{M N}+h_{M N}, B_{M N}=b_{M N}$ around the flat metric $\eta_{M N}$ and zero B-field.

Once again specializing to irreducible couplings one is left with

$$
\beta_{U V}^{a(r)}=B_{a}^{i \Lambda} \widetilde{h}_{i}^{(r)}\left[2^{-\frac{D+n_{\Lambda}+d_{\Lambda}}{2}} B_{b}^{i \Lambda} n^{b} \Sigma_{\Lambda}\left(\frac{\lambda_{U V}^{-1}}{2}\right)+\Gamma^{i \Lambda} \Sigma_{\Lambda}^{\mathrm{e}}\left(\frac{\lambda_{U V}^{-1}}{2}\right)\right] \lambda_{U V}^{-1}
$$

As expected the ultraviolet divergences of irreducible amplitudes are given by a linear combination of the tadpoles $T^{i \Lambda}=2^{-\frac{D+n_{\Lambda}+d_{\Lambda}}{2}} B_{b}^{i \Lambda} n^{b}+\Gamma^{i \Lambda}$ and in a very natural fashion are uniformly cut off in the transverse channel as suggested in [17].

\section{1 "BPS saturated" string amplitudes}

In the following we will consider explicit brane realizations for which the effective gauge theory (2.15) and supergravity (2.21) answers for the $\beta$-function coefficients associated to logarithmically running couplings coincide. We will consider couplings in the $D$-dimensional theory of a finite set of $N$ D-branes probing a background geometry involving generalized D-branes and O-planes. Compact directions for strings with at least one end in the probes can have either DD or ND boundary conditions and therefore $n_{\Lambda}=0$ for all the relevant string excitations. In general, the $\beta$-function coefficients (2.13) and (2.14) receive contributions from infinite towers of open string winding modes or KK-supergravity states, respectively, coming from the reduction on $T^{d}$ of a higher dimensional gauge or supergravity theory. In order to make contact with more familiar $D$-dimensional field theory results we will consider the decompactification limit $R_{j}>>\sqrt{\alpha^{\prime}}$ where the whole tower of open string winding modes decouples $\left(\lim _{R_{j} \rightarrow \infty} W_{d}(t)=1\right)$ and KK supergravity contributions resum to

$$
\begin{aligned}
& \lim _{R_{j} \rightarrow \infty} \frac{P_{d}\left(\frac{\ell}{2}\right)}{v_{d}}=\left(\frac{\ell}{2}\right)^{-\frac{d}{2}} \\
& \lim _{R_{j} \rightarrow \infty} \frac{P_{d}^{e}\left(\frac{\ell}{2}\right)}{v_{d}}=2^{-d}\left(\frac{\ell}{2}\right)^{-\frac{d}{2}}
\end{aligned}
$$

The factor $2^{-d}$ reflects the fact that charge and mass of the higher dimensional Oplanes are democratically distributed between the $2^{d}$ lower dimensional O-planes. Logarithmically running couplings in both the direct and transverse channel then require $d=2$ with $r=\frac{D}{2}$ as the number of boundary insertions. The logarithmic divergences in the open string channel arises as usual from loop integrations of massless gauge particles, while in the supergravity picture they are associated to two-dimensional propagators of massless closed string states. The question one may raise is whether the coefficients in front of the two logarithmic divergences coincide. We will only consider the case with $r$ even e.g. $D=0,4,8$. One can then see that "minimal" supersymmetry in these situations is enough to ensures the agreement 
between the two $\beta$-function coefficients (2.13,2.14). Indeed by assuming the minimal $\mathcal{N}=1$ supersymmetry in $D+2$ dimensions (or equivalently $\mathcal{N}=2$ in $D$ dimensions) the $r=\frac{D}{2}$-point function belongs to the class of "BPS saturated" couplings for which the contributions coming from massive worldsheet bosonic and fermionic states cancel against each other leaving a $t$-independent result.

In order to see this let us consider D3-branes probes $\left(\mathcal{F}^{2}\right.$-term in $\left.D=4\right)$ of $Z_{N}$ orientifold backgrounds. The string partition functions can be written in terms of $Z_{N}$ characters $\chi_{g h}$ defined in terms of the chiral amplitudes $\rho_{g h}$ through

$$
\chi_{g h}(u)=\frac{1}{N} \sum_{l=0}^{N-1} w^{l h} \rho_{g l}(u) \Sigma_{g l}=\operatorname{STr}_{\text {g-twisted }}\left[\sum_{l=0}^{N-1} w^{l h} \Theta^{l} q^{L_{0}-\frac{c}{24}} e^{2 \pi i u_{\mu \nu} J^{\mu \nu}}\right]
$$

where $\Theta$ is the $Z_{N}$ generator, $g, h=0,1, \ldots N-1, \omega=e^{\frac{2 \pi i}{N}}$ and $\Sigma_{g l}$ is the lattice sum in the hyperplane that is fixed under both $g$ and $l$ twists in the $\sigma$ and $\tau$ directions respectively . Expanding (in powers of $u$ ), the helicity supertraces $\rho_{g h}$ in (2.23) for $g, h, Z_{N}$ rotations of a $T^{4}$ torus one can easily check the $t$-independence of the leading orders in the $u$-expansions of (2.23) ("BPS saturated term") ${ }^{4}$

$$
\begin{aligned}
& \rho_{00}(u)=2 \pi u \sum_{\alpha, \beta \neq 0} \eta_{\alpha \beta} \frac{\vartheta\left[\begin{array}{c}
\alpha \\
\beta
\end{array}\right](u \mid \tau)}{\vartheta\left[\begin{array}{l}
\frac{1}{2} \\
\frac{1}{2}
\end{array}\right](u \mid \tau) \eta^{9}}=2 \pi u \frac{\vartheta\left[\begin{array}{c}
\frac{1}{2} \\
\frac{1}{2}
\end{array}\right]^{4}\left(\frac{u}{2} \mid \tau\right)}{\vartheta\left[\begin{array}{c}
\frac{1}{2} \\
\frac{1}{2}
\end{array}\right](u \mid \tau) \eta^{9}}=u^{4}+\ldots \\
& \rho_{0 h}(u)=-8 \pi u \sin ^{2} \frac{\pi h}{N} \sum_{\alpha, \beta \neq 0} \eta_{\alpha \beta} \frac{\vartheta\left[\begin{array}{l}
\alpha \\
\beta
\end{array}\right](u \mid \tau)}{\vartheta\left[\begin{array}{l}
\frac{1}{2} \\
\frac{1}{2}
\end{array}\right](u \mid \tau)} \frac{\vartheta\left[\begin{array}{c}
\alpha \\
\beta
\end{array}\right](0 \mid \tau)}{\eta^{3}} \prod_{e= \pm} \frac{\vartheta\left[\begin{array}{c}
\alpha \\
\beta+e h
\end{array}\right](0 \mid \tau)}{\vartheta\left[\begin{array}{c}
\frac{1}{2} \\
\frac{1}{2}+e h
\end{array}\right](0 \mid \tau)}
\end{aligned}
$$

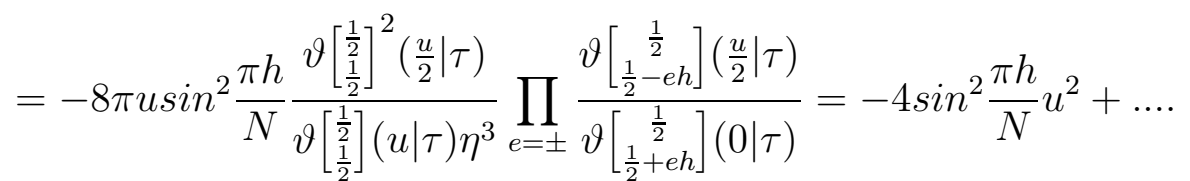

$$
\begin{aligned}
& \rho_{g h}(u)=2 \pi u \sum_{\alpha, \beta \neq 0} \eta_{\alpha \beta} \frac{\vartheta\left[\begin{array}{c}
\alpha \\
\beta
\end{array}\right](u \mid \tau)}{\vartheta\left[\begin{array}{l}
\frac{1}{2} \\
\frac{1}{2}
\end{array}\right](u \mid \tau)} \frac{\vartheta\left[\begin{array}{l}
\alpha \\
\beta
\end{array}\right](0 \mid \tau)}{\eta^{3}} \prod_{e= \pm} \frac{\vartheta\left[\begin{array}{c}
\alpha+e g \\
\beta+e h
\end{array}\right](0 \mid \tau)}{\vartheta\left[\begin{array}{c}
\frac{1}{2}+e g \\
\frac{1}{2}+e h
\end{array}\right](0 \mid \tau)} \\
& =2 \pi u \frac{\vartheta\left[\begin{array}{c}
\frac{1}{2} \\
\frac{1}{2}
\end{array}\right]^{2}\left(\frac{u}{2} \mid \tau\right)}{\vartheta\left[\begin{array}{c}
\frac{1}{2} \\
\frac{1}{2}
\end{array}\right](u \mid \tau) \eta^{3}} \prod_{e= \pm} \frac{\vartheta\left[\begin{array}{c}
\frac{1}{2}-e g \\
\frac{1}{2}-e h
\end{array}\right]\left(\frac{u}{2} \mid \tau\right)}{\vartheta\left[\begin{array}{l}
\frac{1}{2}+e g \\
\frac{1}{2}+e h
\end{array}\right](0 \mid \tau)}=u^{2}+\ldots
\end{aligned}
$$

where dots stand for subleading term in the $u$-expansion. The $t$-independence of the leading order terms in these expansions has already been appreciated in the study of spin effects associated to the interactions of Dp and Dp+4 branes and of anomalous couplings [26]. The result for the $u^{2}$ term in (2.24) is then exact, admitting equivalent SYM $(t \rightarrow \infty)$ and Supergravity $(t \rightarrow 0)$ descriptions, obtained by replacing

\footnotetext{
${ }^{4}$ We borrow the notation and modular transformation properties defined in the appendix A of 25 .
} 
$\left(\rho_{00}, \rho_{0 h}, \rho_{g h}\right)$ with $\left(0,-4 \sin ^{2} \frac{\pi h}{N}, 1\right)$ in the direct and transverse string amplitudes (2.8,2.9). In the decompactification limit (2.22), the $\beta$-function coefficients can then be written in the two alternative forms

$$
\begin{aligned}
& \beta_{I R}^{(2) a}=\frac{1}{4}\left(A_{a b}^{i \Lambda} n^{b}+2 M_{a}^{i \Lambda}\right) h_{i}^{(2)}=\frac{1}{2} \mathrm{l}_{2}\left(\mathcal{R}_{i}^{a}\right) h_{i}^{(2)} \\
& \beta_{U V}^{(2) a}=2\left(2^{-3} B_{b}^{i \Lambda} n^{b}+\frac{1}{4} \Gamma^{i \Lambda}\right) B_{a}^{i \Lambda} h_{i}^{(2)}=2 \hat{T}^{i \Lambda} B_{a}^{i \Lambda} h_{i}^{(2)}
\end{aligned}
$$

with $\operatorname{Tr}_{\mathcal{R}_{i}} T^{a} T^{b}=\mathrm{l}_{2}\left(\mathcal{R}_{i}^{a}\right) \delta^{a b}$ and $\hat{T}^{i \Lambda}=\left(2^{-3} B_{b}^{i \Lambda} n^{b}+\frac{1}{4} \Gamma^{i \Lambda}\right)$ the tadpole associated to the character " $i \Lambda$ " and localized at one of the 4 O7-planes. $t$-independence of the building blocks (2.24) implies exact agreement between the two expressions in (2.25)

$$
\beta_{I R}^{(2) a}=\beta_{U V}^{(2) a}
$$

Indeed plugging the contribution of any $\mathcal{N}=2$ supermultiplet in (2.17), one can easily realize that $h_{i}^{(2)}$ numerically coincides with the Witten index associated to the character " $i$ " of the internal $c=6$ SCFT theory, i.e. $h_{\text {vector }}^{(2)}=-2\left(h_{\text {hyper }}^{(2)}=2\right)$ for $\mathcal{N}=2$ vector (hyper-) multiplets. This integer is, of course, invariant under any modular transformations (in the character basis $S_{i}^{j} h_{j}^{(2)}=h_{i}^{(2)}$ )

As in [1], we are thus allowed to relate direct and transverse channel quantities through the algebraic relations

$$
\begin{aligned}
h_{i}^{(2)} A_{a b}^{i \Lambda} & =h_{i}^{(2)} B_{a}^{i \Lambda} B_{b}^{i \Lambda} \\
h_{i}^{(2)} M_{a}^{i \Lambda} & =h_{i}^{(2)} B_{a}^{i \Lambda} \Gamma^{i \Lambda}
\end{aligned}
$$

which, once inserted in (2.25), lead to a precise matching between IR and UV expressions (2.26).

We conclude that $\mathrm{RG}$-flows induced by $\mathcal{N}=2$ sectors of the gauge theory living on the probe can be accurately described by the linear combination (2.25) of massless tadpoles (one-point functions) $\hat{T}^{i \Lambda}$ in the supergravity theory. Notice that $\mathcal{N}=4$ sectors do not contributes to $h_{i}^{(2)}$ and therefore all relevant contributions share the lattice sum $W_{2}$.

Two comments are in order. First we would like to stress that in establishing the equivalence between $\beta$-functions and tadpoles for brane configurations satisfying the above requirement no large $N$ limit is required. Secondly, as we will see in details in the following, the supersymmetry requirement above allows for $\mathcal{N}=0,1$ brane configurations whose open string excitations can be assembled into sectors that realize different $\mathcal{N}=2$ superalgebras which intersect in an effective less (or non-) supersymmetric gauge theory. Typical examples are D3-branes probing a background of intersecting D7-branes corresponding to F-theory at varying (but weak) coupling. Although the whole effective gauge theory in this case only displays $\mathcal{N}=0,1$ supersymmetry, each sector of the free open string spectrum enjoys $\mathcal{N}=2$ supersymmetry 
which contains the $\mathcal{N}=0,1$ intersection algebra. The cases studied in [3] provide with $\mathcal{N}=0$ examples that fulfill all the above requirements. It would be interesting to test higher-loop corrections to the IR/UV correspondence proposed here for these less supersymmetric brane configurations. In the $\mathcal{N}=1$ examples under study here one would expect that a similar agreement should persist at least at the first subleading $1 / N$ correction to each genus $g$ result. At this order the dominant contributions to the open string amplitudes come from surfaces with no handles and $g+1$ boundaries, $g$ of which are mapped onto the probe D3-branes and only one on the background D7-branes. These give rise to effective $\mathcal{N}=2$ contributions that should not correct the one-loop result. Amplitudes corresponding to surfaces with all the $g+1$ boundaries mapped onto the D3-branes should not contribute to the running because they are associated to $\mathcal{N}=4$ sectors of the open string spectrum [27].

Finally it is instructive to compare the NS-NS tadpoles/RG-flows correspondence under discussion here with the IR/UV correspondence between R-R tadpoles and chiral gauge and gravitational anomalies [1, 19, 28, 29. Indeed the results presented here can be seen as the supersymmetric analogue of the R-R-tadpoles/anomalies correspondence [1]. In that case the topological nature of the interactions allows stronger results irrespective of the presence of spacetime unbroken supersymmetries. Notice that although the results presented here (whenever such IR/UV correspondence exists) are very similar to the anomaly/tadpole correspondence established in [1] the group theory factors weighting the string contributions are obviously different and therefore different are the linear combinations of tadpoles entering the two kinds of computations. In addition the topological index $h_{i}^{(D / 2)}$, numerically equal to the Witten index in $D+2$ dimensions, receives non-trivial contribution in $D$ dimension from the NS sectors in contrast with its anomaly cousin.

\section{D3-branes probes in type II and type IB backgrounds}

In this section we discuss several realizations of the IR/UV correspondence between RG-flows and NS-NS tadpoles for the case of four-dimensional gauge theories living on D3-brane probes of orientifold backgrounds. We will always start by specifying, from the brane data, the open string partition functions for vanishing background, i.e. for $\epsilon_{a b}=0$ in (2.8,2.9), in the direct and transverse channel. In order to simplify the expressions we prefer to display formulas in terms of the amplitudes $\rho_{g h}$ rather than in terms of characters. After translating the resulting amplitudes into the character basis through (2.23) one can check in each case that the Chan-Paton assigments satisfy the consistency conditions (2.10), i.e. that transverse amplitudes factorize as in (2.9) and that the Möbius amplitude is an allowed projection of the Annulus amplitude. The gauge theory will be determined by combining the massless 
contents $\left(q^{0}\right.$-coefficient $)$ of the various partition functions

$$
\begin{aligned}
& \rho_{00}(0)=V+6 O-4 S-4 C+\ldots . \\
& \rho_{0 h}(0)=V+2 O\left(1+2 \cos \frac{2 \pi h}{N}\right)-2(S+C)\left(1+\cos \frac{2 \pi h}{N}\right)+\ldots \\
& \rho_{g h}(0)=2 O-S-C+\ldots
\end{aligned}
$$

where $O, V, S$ and $C$ represent massless four-dimensional scalars, vectors, left- and right- handed spinors respectively.

The $\beta$-function coefficients (2.25) can be easily read from 2.25) with the boundary data $\mathcal{A}_{a b}^{i \Lambda}, \mathcal{M}_{a}^{i \Lambda}, B_{a}^{i}, \Gamma^{i}$ defined through $(2.8,2.9)$ and $h_{i}^{(2)}$ given by the substitutions

$$
\left(\rho_{00}, \rho_{0 h}, \rho_{g h}\right) \rightarrow\left(0,-4 \sin ^{2} \frac{\pi h}{N}, 1\right)
$$

We will denote the $\beta$-function coefficient for the D3 brane gauge group by $\beta_{N}$ with

$$
\beta_{N} \equiv 2 \beta_{I R, U V}^{(2) N}
$$

with $\beta_{I R, U V}^{(2) N}$ given by (2.25). The overall factor of 2 is choosen in such a way to reproduce the familiar $\beta$-function coefficients for gauge group generators normalized as $\operatorname{Tr}_{\text {fund }} Q^{2}=\frac{1}{2}$.

Finally we would like to give a more precise meaning to the linear combination (2.25) of tadpoles generating the RG-flows in the four dimensional gauge theories we will present in the following. As we will see non-trivial contributions to the closed string divergences only come from the $Z_{2}$-string amplitudes $\rho_{01}(0)=V+2 O-4 O+$ massive. We see in particular that no contribution from RR states arise as expected since these states do not couple to $\operatorname{Tr} \mathcal{F}^{2}$. Moreover noticing that the $h^{(2)} \beta$-function coefficients that weighted the string amplitude $\rho_{01}(0)$ satisfies $h_{V+2 O}^{(2)}=-h_{4 O}^{(2)}$ one can identified the linear combination in (2.25) with the tadpole of the ten dimensional dilaton $V+60$. Our results then provide a precise dictionary between this tadpole and $\beta$-function coefficients in the D3-brane gauge theory.

\subsection{D3-branes probing type II D7-backgrounds}

Let us start by considering $N$ D3-branes spanning the 0123 directions as a probe of a background geometry constructed out of two sets of $n_{A}, n_{B}$ intersecting D7branes spanning, besides the spacetime directions common to the D3-branes, the $A=(6789)$ and $B=(4589)$ hyperplanes respectively. We will also introduce the notation $C=(4567)$ for the $\mathrm{D} 7_{A}-\mathrm{D} 7_{B} \mathrm{ND}$ plane. The open string partition function in the direct channel is given by the integral $\int \frac{d t}{t}$ of

$$
\mathcal{A}=t^{-2}\left[\rho _ { 0 0 } \left(N \bar{N} W_{a} W_{b} W_{c}+n_{A} \bar{n}_{A} W_{a} P_{b} P_{c}+n_{B} \bar{n}_{B} P_{a} W_{b} P_{c}\right.\right.
$$

\footnotetext{
${ }^{5}$ For $U(N)$ gauge groups we have an additional identical contribution coming from $\beta_{I R, U V}^{(2) \bar{N}}$
} 


$$
\begin{aligned}
& +\rho_{A 0}\left(N \bar{n}_{A}+n_{A} \bar{N}\right) W_{a}+\rho_{B 0}\left(N \bar{n}_{B}+n_{B} \bar{N}\right) W_{b} \\
& \left.+\rho_{C 0}\left(n_{B} \bar{n}_{A}+n_{A} \bar{n}_{B}\right) P_{c}\right]\left(\frac{i t}{2}\right)
\end{aligned}
$$

where $a, b, c$ stand for the three planes (45), (67) and (89). In the transverse channel one has $\int d \ell$ of

$$
\begin{aligned}
\widetilde{\mathcal{A}}= & 2^{-5}\left[\rho _ { 0 0 } \left(N \bar{N} \frac{P_{a} P_{b} P_{c}}{v_{a} v_{b} v_{c}}+n_{A} \bar{n}_{A} P_{a} W_{b} W_{c} \frac{v_{b} v_{c}}{v_{a}}+n_{B} \bar{n}_{B} W_{a} P_{b} W_{c} \frac{v_{a} v_{c}}{v_{b}}\right.\right. \\
& +\rho_{0 A}\left(N \bar{n}_{A}+n_{A} \bar{N}\right) \frac{P_{a}}{v_{a}}+\rho_{0 B}\left(N \bar{n}_{B}+n_{B} \bar{N}\right) \frac{P_{b}}{v_{b}} \\
& \left.+\rho_{O C}\left(n_{B} \bar{n}_{A}+n_{A} \bar{n}_{B}\right) W_{c} v_{c}\right](i \ell)
\end{aligned}
$$

Expressing (3.4) in terms of the $Z_{2}$-characters (2.23), one can easily check that (3.5) factorizes properly at the origin of the lattice sum and therefore (3.4) defines a sensible CP-charge assignment.

Using the expansion (3.1) one can read the massless spectrum

$$
\begin{array}{ll}
\text { states } & U(N) \times U\left(n_{A}\right) \times U\left(n_{B}\right) \\
V-S-C & (\mathbf{N N}, \mathbf{1}, \mathbf{1})+\left(\mathbf{1}, \mathbf{n}_{\mathbf{A}} \overline{\mathbf{n}}_{\mathbf{A}}, \mathbf{1}\right)+\left(\mathbf{1}, \mathbf{1}, \mathbf{n}_{\mathbf{B}} \overline{\mathbf{n}}_{\mathbf{B}}\right) \\
3(2 O-S-C) & (\mathbf{N} \overline{\mathbf{N}}, \mathbf{1}, \mathbf{1})+\left(\mathbf{1}, \mathbf{n}_{\mathbf{A}} \overline{\mathbf{n}}_{\mathbf{A}}, \mathbf{1}\right)+\left(\mathbf{1}, \mathbf{1}, \mathbf{n}_{\mathbf{B}} \overline{\mathbf{n}}_{\mathbf{B}}\right) \\
(2 O-S-C) & \left(\mathbf{N}, \overline{\mathbf{n}}_{\mathbf{A}}, \mathbf{1}\right)+\left(\mathbf{N}, \mathbf{1}, \overline{\mathbf{n}}_{\mathbf{B}}\right)+\left(\mathbf{1}, \mathbf{n}_{\mathbf{A}}, \overline{\mathbf{n}}_{\mathbf{B}}\right)+\text { h.c. }
\end{array}
$$

The $S U(N) \beta$-function coefficient

$$
\beta_{N}=\left(n_{A}+n_{B}\right)
$$

is always positive, thus leading to an IR free theory on the brane. By setting $n_{A}=0$ or equivalently $n_{B}=0$ one gets a consistent theory with $\mathcal{N}=2$ supersymmetry. The coefficient $\beta_{N}$ can be computed using either (3.4) or (3.5) with the same result, i.e. (3.6).

\subsection{D3-branes in type IB backgrounds}

The next example we will consider is a stack of $N$ D3-branes exploring an $S O\left(n_{A}\right)$ singularity [30]. This background geometry is related to the T-dual version (type IB or simply F-theory at constant coupling [31]) of type I theory on a $T^{2}$ torus with Wilson lines breaking the CP group to $S O(8)^{4}$ [32]. In the case at hand we consider moving $\left(n_{A}-8\right)$ D7-branes far away from one of the 4 O7-planes. The D3-brane theory probes the geometry near this O7-plane. A sensible assigment of CP-charges leads to the open string partition function

$$
\begin{aligned}
\mathcal{A} & =\frac{t^{-2}}{2}\left[\rho_{00}\left(N^{2} W_{a} W_{b} W_{c}+n_{A}^{2} W_{a} P_{b} P_{c}\right)+2 \rho_{A 0} N n_{A} W_{a}\right]\left(\frac{i t}{2}\right) \\
\mathcal{M} & =-\frac{t^{-2}}{2}\left(\rho_{00} n_{A} W_{a} P_{b} P_{c}-\rho_{0 A} N W_{a}\right)\left(\frac{i t}{2}+\frac{1}{2}\right)
\end{aligned}
$$


Rewriting (3.7) in terms of the closed string variables one has $\left(\int d \ell\right)$

$$
\begin{aligned}
\widetilde{\mathcal{A}} & =\frac{2^{-5}}{2}\left[\rho_{00}\left(N^{2} \frac{P_{a} P_{b} P_{c}}{v_{a} v_{b} v_{c}}+n_{A}^{2} P_{a} W_{b} W_{c} \frac{v_{b} v_{c}}{v_{a}}\right)+2 \rho_{0 A} N n_{A} \frac{P_{a}}{v_{a}}\right](i \ell) \\
\widetilde{\mathcal{M}} & =-\frac{2}{2}\left[\rho_{00} n_{A} W_{a}^{\mathrm{e}} W_{b}^{\mathrm{e}} P_{c}^{\mathrm{e}} \frac{v_{a} v_{b}}{v_{c}}+\rho_{0 A} N \frac{P_{a}^{\mathrm{e}}}{v_{a}}\right](i \ell)
\end{aligned}
$$

As expected, the transverse amplitudes factorize properly.

The massless spectrum consists in

$$
\begin{array}{ll}
\text { states } & S p(N) \times S O\left(n_{A}\right) \\
V-S-C & \left(\frac{\mathbf{1}}{2} \mathbf{N}(\mathbf{N}+\mathbf{1}), \mathbf{1}\right)+\left(\mathbf{1}, \frac{\mathbf{1}}{2} \mathbf{n}_{\mathbf{A}}\left(\mathbf{n}_{\mathbf{A}}-\mathbf{1}\right)\right) \\
(2 O-S-C) & \left(\frac{1}{2} \mathbf{N}(\mathbf{N}+\mathbf{1}), \mathbf{1}\right)+\left(\mathbf{1}, \frac{1}{2} \mathbf{n}_{\mathbf{A}}\left(\mathbf{n}_{\mathbf{A}}-\mathbf{1}\right)\right) \\
2(2 O-S-C) & \left(\frac{1}{2} \mathbf{N}(\mathbf{N}-\mathbf{1}), \mathbf{1}\right)+\left(\mathbf{1}, \frac{1}{2} \mathbf{n}_{\mathbf{A}}\left(\mathbf{n}_{\mathbf{A}}-\mathbf{1}\right)\right) \\
(2 O-S-C) & \left(\mathbf{N}, \mathbf{n}_{\mathbf{A}}\right)
\end{array}
$$

The one-loop $\beta$-function coefficient for the $S p(N)$ gauge coupling

$$
\beta_{N}=\frac{1}{2}\left(n_{A}-8\right)
$$

can be made negative (for $n_{A}<8$ ) thanks to the presence of the O7-planes. We thus expect the corresponding theory on the probe to be non-trivial in the IR.

\subsection{D3-branes probes of a $C^{4} / Z_{2}$ Orientifold singularity}

We now start from a T-dual version of the $T^{4} / Z_{2}$ orientifold of type IIB [19, 22, 23 where D9-D5 branes become D3-D7 branes and we send as before the radius of all internal directions to infinity where tadpole conditions can be relaxed. The open string partition function is given by $\left(\int \frac{d t}{t}\right)$

$$
\begin{aligned}
\mathcal{A}= & \frac{t^{-2}}{4}\left[\rho_{00}\left((N+\bar{N})^{2} W_{a} W_{b} W_{c}+\left(n_{A}+\bar{n}_{A}\right)^{2} W_{a} P_{b} P_{c}\right)\right. \\
& -\rho_{0 A}\left((N-\bar{N})^{2}+\left(n_{A}-\bar{n}_{A}\right)^{2}\right) W_{a}+2 \rho_{A 0}(N+\bar{N})\left(n_{A}+\bar{n}_{A}\right) W_{a} \\
& \left.-2 \rho_{A A}(N-\bar{N})\left(n_{A}-\bar{n}_{A}\right) W_{a}\right]\left(\frac{i t}{2}\right) \\
\mathcal{M}= & -\frac{t^{-2}}{4}\left[\rho_{00}\left((N+\bar{N}) W_{a} W_{b} W_{c}+\left(n_{A}+\bar{n}_{A}\right) W_{a} P_{b} P_{c}\right)\right. \\
& \left.-\rho_{0 A}\left(N+\bar{N}+n_{A}+\bar{n}_{A}\right) W_{a}\right]\left(\frac{i t}{2}+\frac{1}{2}\right)
\end{aligned}
$$

Rewritten in terms of the closed string variables one has $\left(\int d \ell\right)$

$$
\begin{aligned}
\widetilde{A}= & \frac{2^{-5}}{4}\left[\rho_{00}\left((N+\bar{N})^{2} \frac{P_{a} P_{b} P_{c}}{v_{a} v_{b} v_{c}}+\left(n_{A}+\bar{n}_{A}\right)^{2} W_{a} W_{b} P_{c} \frac{v_{a} v_{b}}{v_{c}}\right)+\right. \\
& 2 \rho_{0 A}(N+\bar{N})\left(n_{A}+\bar{n}_{A}\right) \frac{P_{a}}{v_{a}}-16 \rho_{A 0}\left((N-\bar{N})^{2}+\left(n_{A}-\bar{n}_{A}\right)^{2}\right) \frac{P_{a}}{v_{a}}
\end{aligned}
$$




$$
\begin{aligned}
& \left.+8 \rho_{A A}(N-\bar{N})\left(n_{A}-\bar{n}_{A}\right) \frac{P_{a}}{v_{a}}\right](i \ell) \\
\widetilde{M}= & -\frac{2}{4}\left[\rho _ { 0 0 } \left(\left((N+\bar{N}) \frac{P_{a}^{\mathrm{e}} P_{b}^{\mathrm{e}} P_{c}^{\mathrm{e}}}{v_{a} v_{b} v_{c}}+\left(n_{A}+\bar{n}_{A}\right) P_{a}^{\mathrm{e}} W_{b}^{\mathrm{e}} W_{c}^{\mathrm{e}} \frac{v_{b} v_{c}}{v_{a}}\right)\right.\right. \\
& \left.+\rho_{0 A}\left(N+\bar{N}+n_{A}+\bar{n}_{A}\right) \frac{P_{a}^{\mathrm{e}}}{v_{a}}\right](i \ell)
\end{aligned}
$$

The massless spectrum is given by

$$
\begin{array}{ll}
\text { states } & U(N) \times U\left(n_{A}\right) \\
V-S-C & (\mathbf{N} \overline{\mathbf{N}}, \mathbf{1})+\left(\mathbf{1}, \mathbf{n}_{\mathbf{A}} \overline{\mathbf{n}}_{\mathbf{A}}\right) \\
(2 O-S-C) & (\mathbf{N} \overline{\mathbf{N}}, \mathbf{1})+\left(\mathbf{1}, \mathbf{n}_{\mathbf{A}} \overline{\mathbf{n}}_{\mathbf{A}}\right) \\
2(2 O-S-C) & \left(\frac{\mathbf{1}}{\mathbf{2}} \mathbf{N}(\mathbf{N}-\mathbf{1}), \mathbf{1}\right)+\left(\mathbf{1}, \frac{\mathbf{1}}{2} \mathbf{n}_{\mathbf{A}}\left(\mathbf{n}_{\mathbf{A}}-\mathbf{1}\right)\right)+\text { h.c. } \\
(2 O-S-C) & \left(\mathbf{N}, \overline{\mathbf{n}}_{\mathbf{A}}\right)+\text { h.c. }
\end{array}
$$

The $S U(N) \beta$-function coefficient at one-loop

$$
\beta_{N}=\frac{1}{2}\left(n_{A}+\bar{n}_{A}-8\right)
$$

receives a negative contribution from the orientifold planes in the closed string description. Notice that a conformal point $n_{A}=\bar{n}_{A}=4$ exists associated to a democratic distribution of the sixteen pairs of D7-branes between the four O7-planes.

\subsection{D3-branes probes of a $C^{6} / Z_{2} \times Z_{2}$ Orientifold singularity}

Finally we will consider $N$ D3 branes in a $T^{6}$ orientifold generated by the group elements $\Omega, Z_{2}^{A}, Z_{2}^{B}$. This is just a T-dual version of the $N=1$ type I background studied in [33]. In addition to the D3-branes and O3-planes, spanning the directions 0123 common to all the branes, three groups of D7-branes and O7-planes are require to invade the $A=$ (6789), $B=$ (4589) and $C=$ (4567) hyperplanes respectively. String amplitudes $\rho_{g h}$ and $Z_{2} \times Z_{2}$ characters are related to each other by

$$
\left(\begin{array}{c}
\chi_{g 0} \\
\chi_{g A} \\
\chi_{g B} \\
\chi_{g C}
\end{array}\right)=S_{S O(8)}\left(\begin{array}{c}
\rho_{g 0} \Sigma_{g 0} \\
\rho_{g A} \Sigma_{g A} \\
\rho_{g B} \Sigma_{g B} \\
\rho_{g C} \Sigma_{g C}
\end{array}\right)=\frac{1}{2}\left(\begin{array}{cccc}
+ & + & + & + \\
+ & + & - & - \\
+ & - & - & + \\
+ & - & + & -
\end{array}\right)\left(\begin{array}{c}
\rho_{g 0} \Sigma_{g 0} \\
\rho_{g A} \Sigma_{g A} \\
\rho_{g B} \Sigma_{g B} \\
\rho_{g C} \Sigma_{g C}
\end{array}\right)
$$

which generalizes (2.23) to this case. The open string partition function in the direct channel reads [33] $\left(\int \frac{d t}{t}\right)$

$$
\begin{aligned}
\mathcal{A}= & \frac{t^{-2}}{2}\left[\rho_{00}\left(N^{2} W_{a} W_{b} W_{c}+n_{A}^{2} W_{a} P_{b} P_{c}+n_{B}^{2} P_{a} W_{b} P_{c}+n_{C}^{2} P_{a} P_{b} W_{c}\right)\right. \\
& +2 \rho_{A 0}\left(N n_{A} W_{a}+n_{B} n_{C} P_{a}\right)+2 \rho_{B 0}\left(N n_{B} W_{b}+n_{A} n_{C} P_{b}\right) \\
& \left.+2 \rho_{C 0}\left(N n_{C} W_{c}+n_{A} n_{B} P_{c}\right)\right]\left(\frac{i t}{2}\right)
\end{aligned}
$$




$$
\begin{aligned}
\mathcal{M}= & \frac{t^{-2}}{2}\left[-\frac{1}{2} \rho_{00}\left(N W_{a} W_{b} W_{c}+n_{A} W_{a} P_{b} P_{c}+n_{B} P_{a} W_{b} P_{c}+n_{C} P_{a} P_{b} W_{c}\right)\right. \\
& +\frac{1}{2} \rho_{0 A}\left(\left(N+n_{A}\right) W_{a}+\left(n_{B}+n_{C}\right) P_{a}\right)+\frac{1}{2} \rho_{0 B}\left(\left(N+n_{B}\right) W_{b}+\left(n_{A}+n_{C}\right) P_{b}\right) \\
& \left.+\frac{1}{2} \rho_{0 C}\left(\left(N+n_{C}\right) W_{c}+\left(n_{A}+n_{B}\right) P_{c}\right)\right]\left(\frac{i t}{2}+\frac{1}{2}\right)
\end{aligned}
$$

Once rewritten in terms of the closed string variables, (3.14) yields $\left(\int d \ell\right)$

$$
\begin{aligned}
\widetilde{\mathcal{A}}= & \frac{2^{-4}}{4}\left[\rho_{00}\left(N^{2} \frac{P_{a} P_{b} P_{c}}{v_{a} v_{b} v_{c}}+n_{A}^{2} P_{a} W_{b} W_{c} \frac{v_{b} v_{c}}{v_{a}}+n_{B}^{2} W_{a} P_{b} W_{c} \frac{v_{a} v_{c}}{v_{b}}+n_{C}^{2} W_{a} W_{b} P_{c} \frac{v_{a} v_{b}}{v_{c}}\right)\right. \\
& +2 \rho_{0 A}\left(N n_{A} \frac{P_{a}}{v_{a}}+n_{B} n_{C} W_{a} v_{a}\right)+2 \rho_{0 B}\left(N n_{B} \frac{P_{b}}{v_{b}}+n_{A} n_{C} W_{b} v_{b}\right) \\
& \left.+2 \rho_{O C}\left(N n_{C} \frac{P_{c}}{v_{c}}+n_{A} n_{B} W_{c} v_{c}\right)\right](i \ell) \\
\widetilde{\mathcal{M}}= & -\frac{2}{4}\left[\rho_{00}\left(N \frac{P_{a}^{\mathrm{e}} P_{b}^{\mathrm{e}} P_{c}^{\mathrm{e}}}{v_{a} v_{b} v_{c}}+n_{A} P_{a}^{\mathrm{e}} W_{b}^{\mathrm{e}} W_{c}^{\mathrm{e}} \frac{v_{c} v_{b}}{v_{a}}+n_{B} W_{a}^{\mathrm{e}} P_{b}^{\mathrm{e}} W_{c}^{\mathrm{e}} \frac{v_{a} v_{c}}{v_{b}}+n_{C} W_{a}^{\mathrm{e}} W_{b}^{\mathrm{e}} P_{c}^{\mathrm{e}} \frac{v_{b} v_{a}}{v_{c}}\right)\right. \\
& +\rho_{0 A}\left(\left(N+n_{A}\right) \frac{P_{a}^{\mathrm{e}}}{v_{a}}+\left(n_{B}+n_{C}\right) W_{a}^{\mathrm{e}} v_{a}\right)+\rho_{0 B}\left(\left(N+n_{B}\right) \frac{P_{b}^{\mathrm{e}}}{v_{b}}+\left(n_{A}+n_{C}\right) W_{b}^{\mathrm{e}} v_{b}\right) \\
& \left.+\rho_{O C}\left(\left(N+n_{C}\right) \frac{P_{c}^{\mathrm{e}}}{v_{c}}+\left(n_{A}+n_{B}\right) W_{c}^{\mathrm{e}} v_{c}\right)\right](i \ell)
\end{aligned}
$$

Once again, one can check that the transverse amplitudes factorize properly. In particular for the states with vanishing winding and momentum one finds a sum of perfect squares for $\widetilde{\mathcal{A}}$ and a compatible sum of products for $\widetilde{\mathcal{M}}$.

The massless spectrum reads

$$
\begin{array}{ll}
\text { states } & S p(N) \times S p\left(n_{A}\right) \times S p\left(n_{B}\right) \times S p\left(n_{C}\right) \\
V-S-C & \left(\frac{\mathbf{1}}{\mathbf{2}} \mathbf{N}(\mathbf{N}+\mathbf{1}), \mathbf{1}, \mathbf{1}, \mathbf{1}\right)+\left(\mathbf{1}, \frac{\mathbf{1}}{\mathbf{2}} \mathbf{n}_{\mathbf{A}}\left(\mathbf{n}_{\mathbf{A}}+\mathbf{1}\right), \mathbf{1}, \mathbf{1}\right) \\
& \left(\mathbf{1}, \mathbf{1}, \frac{\mathbf{1}}{\mathbf{2}} \mathbf{n}_{\mathbf{B}}\left(\mathbf{n}_{\mathbf{B}}+\mathbf{1}\right), \mathbf{1}\right)+\left(\mathbf{1}, \mathbf{1}, \mathbf{1}, \frac{\mathbf{1}}{\mathbf{2}} \mathbf{n}_{\mathbf{C}}\left(\mathbf{n}_{\mathbf{C}}+\mathbf{1}\right)\right) \\
3(2 O-S-C) & \left(\frac{\mathbf{1}}{\mathbf{2}}(\mathbf{N}-\mathbf{1}), \mathbf{1}, \mathbf{1}, \mathbf{1}\right)+\left(\mathbf{1}, \frac{\mathbf{1}}{\mathbf{2}} \mathbf{n}_{\mathbf{A}}\left(\mathbf{n}_{\mathbf{A}}-\mathbf{1}\right), \mathbf{1}, \mathbf{1}\right) \\
& \left(\mathbf{1}, \mathbf{1}, \frac{\mathbf{1}}{\mathbf{2}} \mathbf{n}_{\mathbf{B}}\left(\mathbf{n}_{\mathbf{B}}-\mathbf{1}\right), \mathbf{1}\right)+\left(\mathbf{1}, \mathbf{1}, \mathbf{1}, \frac{\mathbf{1}}{\mathbf{2}} \mathbf{n}_{\mathbf{C}}\left(\mathbf{n}_{\mathbf{C}}-\mathbf{1}\right)\right) \\
& \left(\mathbf{N}, \mathbf{n}_{\mathbf{A}}, \mathbf{1}, \mathbf{1}\right)+\left(\mathbf{N}, \mathbf{1}, \mathbf{n}_{\mathbf{B}}, \mathbf{1}\right)+\left(\mathbf{N}, \mathbf{1}, \mathbf{1}, \mathbf{n}_{\mathbf{C}}\right) \\
& \left(\mathbf{1}, \mathbf{n}_{\mathbf{A}}, \mathbf{n}_{\mathbf{B}}, \mathbf{1}\right)+\left(\mathbf{1}, \mathbf{n}_{\mathbf{A}}, \mathbf{1}, \mathbf{n}_{\mathbf{C}}\right)+\left(\mathbf{1}, \mathbf{1}, \mathbf{n}_{\mathbf{B}}, \mathbf{n}_{\mathbf{C}}\right)
\end{array}
$$

Depending on the number of background D7-branes of various kinds, the $S p(N)$ $\beta$-function coefficient

$$
\beta_{N}=\frac{1}{2}\left(n_{A}+n_{B}+n_{C}-12\right)
$$

can be vanishing, positive or negative. A (super)conformal four dimensional gauge theory is found for $n_{A}+n_{B}+n_{C}=12$.

\section{AdS/CFT correspondence and final comments}

Soon after Maldacena's proposal of identifying type IIB superstring on $A d S_{5} \times S^{5}$ 
with $N$ R-R 5 -form fluxes and $\mathcal{N}=4 \mathrm{SYM}$ with gauge group $S U(N)$ [34], many other candidate supergravity duals of superconformal field theories in $D=4$ have been proposed [35]. In particular D3-branes exploring F-theory backgrounds at constant coupling were identified as a natural arena for the holographic study of superconformal field theories [6]. The near horizon geometry of a stack of $N$ D3-branes in an F-theory background at constant imaginary coupling is given by

$$
\begin{aligned}
d s^{2} & =H(y)^{-1 / 2} \eta_{\mu \nu} d x^{\mu} d x^{\nu}+H(y)^{1 / 2} g_{i j} d y^{i} d y^{j} \\
e^{\phi} & =g_{s} \\
F_{5} & =N\left(\epsilon_{5}+* \epsilon_{5}\right) .
\end{aligned}
$$

It locally looks like $A d S_{5} \times E_{5}$, where $E_{5}$ is some Einstein manifold that replaces the familiar $S^{5}$ [36, 37]. The scale of the cosmological constants of the two factors is set by $L^{4}=g_{s} N\left(\alpha^{\prime}\right)^{2}, \epsilon_{5}$ is the $A d S_{5}$ volume form and $H$ is a harmonic function in the Ricci flat metric $g_{i j}$ transverse to the 3-branes. All other fields are set to zero in the background.

We are interested in the cases when the mutually non-local 7-branes are grouped in such a way as to give rise to bound-states of D7-branes and O7-planes at constant weak coupling [31]. These configurations admit a perturbative description in terms of open and unoriented strings. The metric of the transverse space is explicitly known to be [6]

$$
d r^{2}+r^{2} d s_{5}^{2}=\sum_{a=1}^{3} \frac{\left|d z_{a}\right|^{2}}{\left|z_{a}\right|^{\alpha_{a}}}
$$

with $r^{2}=\sum_{a=1}^{3}\left|w_{a}\right|^{2}$ and $w_{a}=z_{a}^{1-\frac{\alpha_{a}}{2}}$. The exponent $\alpha_{a}$ is 1 (0) when there are (no) 7-branes invading the complex plane labeled by $a$.

After performing two T-dualities along directions transverse to the 3-branes, the above F-theory background is mapped to a perturbative type I background. In the previous sections, we have shown that the factorization of unoriented/open string vacuum amplitudes in the presence of background gauge fields allows one to deduce the coupling of the bulk closed string states and in principle to reconstruct the metric, dilaton and p-form backgrounds.

Following [3] and relying on the results of the previous sections, we now turn to consider cases in which the dilaton varies in the F-theory background. Indeed by displacing some of the D7-branes from the orientifold planes it is possible to generate a local tadpole for the 10-D dilaton on the disk and the projective plane. In the large $N$ counting these contributions are subleading and are holographically dual to corrections that drive a logarithmic running of the gauge coupling in theories that are (super)conformal in the large $N$ limit. The expansion in powers of $1 / N$ is systematic and the coupled system of equations admits a solution that is asymptotic to $A d S_{5} \times E_{5}$. 
The relevant system of equations has been studied by [3]. We will perform a similar first-order analysis for more general F-theory-like configurations with "dilaton" tadpoles. Since the R-R axion and the complex antisymmetric tensor appear at least quadratically in the equations of motion for the metric, dilaton and four-index antisymmetric tensor it is consistent for our purposes to truncate the theory to the latter set of fields. In the presence of tadpoles, the relevant equations of motion read

$$
\begin{array}{ll}
e^{-2 \phi} & {\left[8(\nabla \phi)^{2}-8 \nabla^{2} \phi-2 R\right]=e^{-\phi} T^{\phi}+\ldots} \\
e^{-2 \phi} & {\left[R_{M N}+2 \nabla_{M} \nabla_{N} \phi\right]-\frac{1}{96}\left[F_{M P Q R S} F_{N} P Q R S-\frac{1}{10} g_{M N} F_{P Q R S T} F^{P Q R S T}\right]} \\
& =e^{-\phi} T_{M N}^{g}+\ldots \\
\nabla^{M} & F_{M N P Q R}=T_{N P Q R}^{A}-\ldots
\end{array}
$$

Notice the absence of the suppression factor $e^{+\phi_{o}}=g_{s} \approx 1 / N$ in front of the RRtadpole in the last equation. The $1 / N$ suppression comes in this case from the overall scaling of the background self-dual five-form with $N$.

Starting from the solution (4.1) of the above equations of motion at vanishing tadpoles, one can turn on the tadpoles and linearize the system around (4.1). An ansatz for the metric and the 10-D dilaton that is compatible with four-dimensional Poincaré invariance is

$$
\begin{aligned}
d s^{2} & =d \tau^{2}+e^{2 \lambda(\tau)} \eta_{\mu \nu} d x^{\mu} d x^{\nu}+e^{2 \nu(\tau)} \hat{g}_{\alpha \beta} d \theta^{\alpha} d \theta^{\beta} \\
\phi & =\phi(\tau)
\end{aligned}
$$

where $\hat{g}_{\alpha \beta}$ is the constant curvature metric of the Einstein space $E_{5}$ defined by (4.2). To the order at which we are going to work the correction to the R-R fields, including the four-form potential, will play no role in the evolution of the dilaton. This can be easily seen using the traceless property of the stress energy tensor associated to a four form RR-potential in order to eliminate the curvature scalar from the dilaton equation. Indeed barring the fields with vanishing background, the scalar curvature is entirely given in terms of the dilaton by

$$
R=-2 \nabla^{2} \phi-\frac{5}{2} T^{\phi} e^{+\phi}
$$

Plugging $R$ into the equation of motion for the dilaton yields

$$
8(\nabla \phi)^{2}-4 \nabla^{2} \phi=4 T^{\phi} e^{+\phi}
$$

that combined with the ansatz (4.5) for the dilaton and the metric leads to

$$
\ddot{\phi}+(4 \dot{\lambda}+5 \dot{\nu}) \dot{\phi}-2(\dot{\phi})^{2}=T^{\phi} e^{+\phi}
$$

where a dot denotes a derivative with respect to the radial transverse coordinate $\tau$. Eq. (4.8) agrees with the corresponding equation derived in [3] for the simpler cases with vanishing $\mathrm{R}-\mathrm{R}$ tadpoles up to the overall factor in the source term. 
Expanding with respect to the solution with $e^{\phi_{o}}=g_{s}, \lambda_{o}=\tau / L$ and $e^{\nu_{o}}=L^{2}$ for vanishing tadpoles

$$
\begin{aligned}
\phi & =\phi_{o}+\frac{1}{N} \phi_{1}+\ldots \\
\lambda & =\lambda_{o}+\frac{1}{N} \lambda_{1}+\ldots \\
\nu & =\nu_{o}+\frac{1}{N} \nu_{1}+\ldots
\end{aligned}
$$

one immediately sees that $\phi_{1}$ decouples from the remaining fields $\lambda_{1}$ and $\nu_{1}$, since $\dot{\phi}_{o}=0$. Using $\dot{\lambda}_{o}=1 / L$ we are left with

$$
\ddot{\phi}_{1}+\frac{4}{L} \dot{\phi}_{1}=4 T^{\phi}
$$

that admits $\phi_{1}=T_{\phi} L \tau$ as an obvious solution. After a proper definition of the radial variable $u=\int e^{\lambda(\tau)} d \tau \approx L e^{\tau / L}$ the correction to the dilaton turns out to be logarithmic in $u$ with $\beta$-function coefficient $T_{\phi}$ as in the case studied by [3]. Notice that it is the $10-\mathrm{D}$ dilaton $\phi$ that has to be holographically identified with the gauge coupling [14].

We interpret this as a logarithmic running of the coupling constant in the boundary gauge theory. Notice that our argument above has the virtue to establishing a precise identification of the one-loop $\beta$-function coefficient with the 10-D dilaton tadpole. Being the dilaton tadpole associated to a UV divergence we expect it to be independent of the large distance properties of the background and we find no need to perform a fullfledged string computation around $A d S_{5}$ but rather use the flat-spacetime results of the previous sections.

In the cases that we have explicitly studied the coefficient can be negative only when orientifold planes are present: brane configurations tend to have positive $\beta$ function and thus to expose a trivial IR behaviour. We do not know whether this is a serious drawback for the purposes of describing non super-conformal gauge theories in terms of brane probes or simply a limitation imposed by the configurations we have considered.

Moreover we also don't know whether two- and higher loop contributions can spoil the picture especially when supersymmetry is broken and the scalar self-couplings tend to run in the wrong direction. Clearly further study is needed. Extracting the $\beta$-function coefficient from a genuine two-loop computation in theories of open and unoriented strings seems feasable and we hope to return to this issue in the near future.

Although higher loops might show a systematic pattern [38, 39 the world-sheet duality between open and closed string channels gets more involve. Nevertheless it 
can prove to be an unprecedented tool in the interplay between gauge theory and gravity: a bridge over trouble waters.

\section{Acknowledgements}

We would like to acknowledge fruitful discussions with C. Angelantonj, A. Armoni, D. Freedman, M. Green, S. Kovacs, J. Minahan, G. Pradisi, G. Rossi, A. Sagnotti and Ya. Stanev. This work was in part supported by the EEC contract HPRN-CT2000-00122 and the INTAS project 991590.

\section{References}

[1] M. Bianchi and J.F. Morales, JHEP 0003 (2000) 030, hep-th/0002149

[2] W. Fischler and L. Susskind, Phys. Lett. B171 (1986) 383; ibid. B173 (1986) 262.

[3] C. Angelantonj and A. Armoni, "Non-Tachyonic Type OB Orientifolds, NonSupersymmetric Gauge Theories and Cosmological RG Flow", hep-th/9912257; " $R G$ Flow, Wilson Loops and the Dilaton Tadpole", hep-th/0003050.

[4] A. Sagnotti, Nucl. Phys. Proc. Suppl. 56B (1997) 332 hep-th/9702093.

[5] R. Blumenhagen, A. Font and Dieter Lüst, Nucl. Phys. B558 (1999) 159, hepth/9904069; Nucl. Phys. B560 (1999) 66, hep-th/9906101]; G. Aldazabal and A. M. Uranga, JHEP 9910 (1999) 024 [hep-th/9908072].

[6] O. Aharony, A. Fayyazuddin and J. Maldacena, JHEP 9807 (1998) 013 hepth/9806159].

[7] I. R. Klebanov and A. A. Tseytlin, Nucl. Phys. B546 (1999) 155, hep-th/9811035; Nucl. Phys. B547 (1999) 143, hep-th/9812089; JHEP 9903 (1999) 015, hepth/9901101].

[8] J. A. Minahan, JHEP 9904 (1999) 007 [hep-th/9902074].

[9] L. Girardello, M. Petrini, M. Porrati and A. Zaffaroni, JHEP 9812 (1998) 022 hepth/9810126; JHEP 9905 (1999) 026 hep-th/9903026; Nucl. Phys. B569 (2000) 451 [hep-th/9909047.

[10] D. Z. Freedman, S. S. Gubser, K. Pilch and N. P. Warner, "Renormalization group flows from holography supersymmetry and a c-theorem," hep-th/9904017; "Continuous distributions of D3-branes and gauged supergravity," hep-th/9906194.

[11] J. Distler and F. Zamora, Adv. Theor. Math. Phys. 2 (1999) 1405 hep-th/9810206]; JHEP 0005 (2000) 005 [hep-th/9911040].

[12] O. DeWolfe and D. Z. Freedman, "Notes on fluctuations and correlation functions in holographic renormalization group flows," hep-th/0002226; G. Arutyunov, S. Frolov 
and S. Theisen, "A note on gravity-scalar fluctuations in holographic $R G$ flow geometries," hep-th/0003116.

[13] C. V. Johnson, A. W. Peet and J. Polchinski, Phys. Rev. D61 (2000) 086001 hepth/9911161]; J. Polchinski and M. J. Strassler, "The string dual of a confining fourdimensional gauge theory", hep-th/0003136.

[14] K. Pilch and N. P. Warner, " $N=2$ supersymmetric $R G$ flows and the IIB dilaton", hep-th/0004063; " $N=1$ supersymmetric renormalization group flows from IIB supergravity", hep-th/0006066.

[15] E. Alvarez and C. Gomez, JHEP 0005 (2000) 012 hep-th/0003205; Phys. Lett. B476 (2000) 411 [hep-th/0001016]; Nucl. Phys. B574 (2000) 153 hep-th/9911215]; JHEP 9910 (1999) 018 hep-th/9907205; Nucl. Phys. B566 (2000) 363 hepth/9907158; Nucl. Phys. B550 (1999) 169 hep-th/9902012].

[16] C. Bachas and C. Fabre, Nucl. Phys. B476 (1996)418, hep-th/9605028.

[17] I. Antoniadis, C. Bachas, E. Dudas Nucl. Phys. B560 (1999)93, hep-th/9906039.

[18] A. Sagnotti, in Non Perturbative Quantum Field Theory, ed. G. Mack et al. (Pergamon, New York, 1988) 521; M. Bianchi, G. Pradisi and A. Sagnotti, Phys. Lett. B273 (1991) 389; D. Fioravanti, G. Pradisi and A. Sagnotti, Phys. Lett. B321 (1994) 349; G. Pradisi, A. Sagnotti, Ya.S. Stanev, Phys. Lett. B354 (1995) 279; B356 (1995) 230; B381 (1996) 97, [hep-th/9603097].

[19] M. Bianchi and A. Sagnotti, Phys. Lett. B247 (1990) 517; Nucl. Phys. B361 (1991) 519.

[20] C. Bachas and M. Porrati, Phys. Lett. B296 (1992) 77 hep-th/9209032; C. Bachas, in String theory, quantum gravity and the unification of fundamental interactions, ed. M. Bianchi et al. (World Scientific, Singapore 1993) 16.

[21] A. Abouelsaood, C. G. Callan, C. R. Nappi and S. A. Yost, Nucl. Phys. B280 (1987) 599.

[22] G. Pradisi and A. Sagnotti, Phys. Lett. B216 (1989) 59.

[23] E.G. Gimon and J. Polchinski, Phys. Rev. D54 (1996) 1667, hep-th/9601038.

[24] M. B. Green and M. Gutperle, Nucl. Phys. B476 (1996)484, hep-th/9604091.

[25] M. Bianchi, J. F. Morales and G. Pradisi, Nucl. Phys. B573 (2000) 314, hepth/9910228].

[26] J. F. Morales, C. A. Scrucca and M. Serone, Nucl. Phys. B534 (1998) 223 hep-th/9801183; Nucl. Phys. B552 (1999) 291 hep-th/9812071; M. Serone, J. F. Morales, J. C. Plefka, C. A. Scrucca and A. K. Waldron, "Spin dependent D-brane interactions and scattering amplitudes in matrix theory", hep-th/9812039. 
[27] M. Bershadsky, Z. Kakushadze and C. Vafa, Nucl. Phys. B523 (1998) 59 hepth/9803076]; A. Lawrence, N. Nekrasov and C. Vafa, Nucl. Phys. B533 (1998) 199 [hep-th/9803015.

[28] Y. Cai and J. Polchinski, Nucl. Phys. B296 (1988) 91.

[29] G. Aldazabal, D. Badagnani, L. E. Ibanez and A. M. Uranga, JHEP 9906 (1999) 031 hep-th/9904071.

[30] T. Banks, M.R. Douglas and N. Seiberg, Phys. Lett. B387 (1996)278, hepth/9605199.

[31] A. Sen, Phys. Rev. D55 (1997) 7345 [hep-th/9702165].

[32] M. Bianchi, G. Pradisi and A. Sagnotti, Nucl. Phys. B376 (1992) 365.

[33] M. Berkooz and R. G. Leigh, Nucl. Phys. B483 (1997) 187, hep-th/9605049]; C. Angelantonj, I. Antoniadis, G. D'Appollonio, E. Dudas and A. Sagnotti, Nucl. Phys. B572 (2000)36, hep-th/9911081.

[34] J.M. Maldacena, Adv. Theor. Math. Phys. 2 (1998) 231; Int. J. Theor. Phys. 38 (1999) 1113, hep-th/9711200.

[35] O. Aharony, S. S. Gubser, J. Maldacena, H. Ooguri and Y. Oz, Phys. Rept. 323 (2000) 183 [hep-th/9905111.

[36] I. R. Klebanov and E. Witten, Nucl. Phys. B556 (1999) 89 hep-th/9905104; Nucl. Phys. B536 (1998) 199 hep-th/9807080.

[37] A. Kehagias, Phys. Lett. B435 (1998) 337 hep-th/9805131].

[38] J. Khoury and H. Verlinde, "On Open/Closed String Duality", hep-th/0001056

[39] J. de Boer, E. Verlinde and H. Verlinde, On the Holographic Renormalization Group", hep-th/9912012. 